\title{
An Explicit Expression for the Wavelength of a Gravity Wave
}

\author{
F. C. W. OLson \\ Naval Coastal Systems Laboratory, Panama City, Fla. 32401 \\ 6 October 1972 and 20 November 1972
}

\section{ABSTRACT}

\begin{abstract}
The square of the wavelength $\lambda$ of a surface gravity wave is expressed as an eighth-degree polynomial in $u=4 \pi^{2} h /\left(g T^{2}\right)$, where $h$ is the depth and $T$ the period. The relative error in $\lambda / \lambda_{0}$, where $\lambda_{0}$ is the deep water wavelength, is less than $0.0004 \%$ for $u<1$ and less than $1 \%$ for $u<2$.
\end{abstract}

\section{Introduction}

The wavelength $\lambda$, period $T$, and water depth $h$ for a surface gravity wave, based on linear theory, are related by

$$
\sigma^{2}=g k \tanh k h,
$$

where

$$
\left.\begin{array}{l}
\sigma=2 \pi / T \\
k=2 \pi / \lambda
\end{array}\right\}
$$

and $g$ is the acceleration due to gravity. The nonlinear manner in which the wavelength appears in (1) has lead to various stratagems for determining the wavelength. Perhaps the best known is the two-table method used by Wiegel (1964). This is well suited for most practical applications.

In carrying out some theoretical studies, the writer found a need for an explicit expression of the wavelength in terms of the depth and wave period. The purpose of this note is to derive such an expression. 


\section{Derivation}

We write (1) as

$$
\frac{\sigma^{2}}{g k}=\frac{k_{0}}{k}=\tanh k h .
$$

It is easy to show that $k_{0}$ is the deep water wavenumber. If we let $k_{0} / k=x,(2)$ can be written

$$
k h=\tanh ^{-1} x=x+\frac{x^{3}}{3}+\frac{x^{5}}{5}+\cdots,
$$

or

$$
k_{0} h=z+\frac{z^{2}}{3}+\frac{z^{3}}{5}+\cdots
$$

where $z=x^{2}$. We now reverse the series (4) using Formula 159 in Jolley (1961). Thus,

and

$$
y=k_{0} h=z-b_{1} z^{2}-b_{2} z^{3}-\cdots,
$$

where

$$
z=y+C_{1} y^{2}+C_{2} y^{3}+\cdots,
$$

$$
\left.\begin{array}{l}
C_{1}=b_{1} \\
C_{2}=b_{2}+2 b_{1}^{2} \\
C_{3}=b_{3}+5 b_{1} b_{2}+5 b_{1}^{3} \text {, etc. }
\end{array}\right\} .
$$

Thus, Eq. (6) may be written

$$
z=x^{2}=k_{0} h-\frac{1}{3}\left(k_{0} h\right)^{2}+\frac{1}{45}\left(k_{0} h\right)^{3}+\frac{1}{189}\left(k_{0} h\right)^{4}+\cdots,
$$

\begin{tabular}{|c|c|c|c|}
\hline \multirow[b]{2}{*}{$u$} & \multicolumn{2}{|c|}{$\lambda / \lambda_{0}$} & \multirow{2}{*}{$\begin{array}{c}\text { Relative } \\
\text { error } \\
(\%)\end{array}$} \\
\hline & Eq. (1) & Eq. (8) & \\
\hline $\begin{array}{l}0.5 \\
1.0 \\
1.5 \\
2.0 \\
2.5 \\
3.0\end{array}$ & $\begin{array}{l}0.647918229 \\
0.833556559 \\
0.924887611 \\
0.96836 \\
0.98743 \\
0.99520\end{array}$ & $\begin{array}{l}0.64791836 \\
0.83355996 \\
0.9248819 \\
0.9676 \\
0.9787 \\
0.9386\end{array}$ & $\begin{array}{l}\quad 0.00002 \\
0.0004 \\
-0.0006 \\
-0.076 \\
-0.89 \\
-5.7\end{array}$ \\
\hline
\end{tabular}

or in nondimensional form with the deep water wavelength $\lambda_{0}=g T^{2} /(2 \pi)$ and $u=\sigma^{2} h / g$,

$$
\begin{aligned}
\left(\lambda / \lambda_{0}\right)^{2}= & u-\frac{1}{3} u^{2}+\frac{1}{45} u^{3}+\frac{1}{189} u^{4} \\
& +0.000776014 u^{5}-0.000044892 u^{6} \\
& -0.000071391 u^{7}-0.000022654 u^{8}+\cdots
\end{aligned}
$$

TABLe 1. Comparison of wavelengths computed with Eqs. (1) and (8).

This completes the formal solution. The wavelength $\lambda$ is now given explicitly in terms of the depth $h$ and the period $T$.

The question of the convergence of (8) is unresolved and perhaps will remain so for a long time since an expression for the general term $C_{n}$ does not seem to be known. Since we will be dealing with only a finite number of terms in (8), convergence in a strict mathematical sense will have little meaning. The question is rather, how well does (8) predict the wavelength implied by (1)?

\section{Numerical verification}

On examining (8), it is reasonable to suspect that as long as $u<1$, wavelengths predicted by (8) will be fairly accurate. The range of validity for $u>1$ can be determined by trial. Table 1 gives $\lambda / \lambda_{0}$ computed by (1) and by (8) for $u=0.5,1,1.5,2.0,2.5$ and 3.0 , together with the relative error.

In interpreting Table 1 it is helpful to know that the eighth-degree polynomial in (8) has a maximum at $u=2.4176$ and that $\lambda$ computed with this value of $u$ is $97.9 \%$ of $\lambda_{0}$.

\section{REFERENCES}

Jolley, L. B. W., 1961 : Summation of Series. New York, Dover, 251 pp.

Wiegal, R. I., 1964: Oceanographical Engineering. Englewood Cliffs, N. J., Prentice Hall, 532 pp. 